\title{
The minimum Jeans mass, brown dwarf companion IMF, and predictions for detection of Y-type dwarfs
}

\author{
B. Zuckerman ${ }^{1}$ and I. Song ${ }^{2}$ \\ 1 Dept. of Physics \& Astronomy and Center for Astrobiology, University of California, Los Angeles, 475 Portola Plaza, Los Angeles, \\ CA 90095-1547, USA \\ e-mail: ben@astro.ucla.edu \\ 2 Department of Physics \& Astronomy, The University of Georgia, Athens, GA 30605, USA \\ e-mail: song@physast .uga . edu \\ Received 23 April 2008 / Accepted 14 November 2008 \\ ABSTRACT

\begin{abstract}
Cool L- and T-type objects were discovered first as companions to stars in 1988 and 1995, respectively. A certain example of the even cooler Y-type spectral class $\left(T_{\text {eff }} \lessgtr 500 \mathrm{~K}\right)$ has not been seen. Recent infrared-imaging observations of stars and brown dwarfs indicate that substellar companions with large semi-major axes and with masses less than the brown dwarf/giant planet dividing line $\left(\sim 13.5 M_{\mathrm{J}}\right)$ are rare. Theoretical considerations of the Jeans mass fragmentation of molecular clouds are consistent with this minimum mass cutoff and also with the semi-major axis (hundreds of AU) characteristic of the lowest mass imaged companions. As a consequence, Y-class companions with large semi-major axes should be scarce around stars $<2 \mathrm{Gyr}$ old, and also around substellar primaries of all ages. By focusing on brown dwarf companions to young stellar primaries, it is possible to derive a first estimate of the brown dwarf IMF over the entire range of brown dwarf masses $\left(13 M_{\mathrm{J}}\right.$ to $\left.79 M_{\mathrm{J}}\right)$ - the number of companion brown dwarfs is proportional to the mass to the $-1.2 \pm 0.2$ power.
\end{abstract}

Key words. stars: planetary systems - stars: low-mass, brown dwarfs

\section{Introduction}

The temperature of the coolest measured substellar dwarf objects has diminished from $1900 \mathrm{~K}$ in 1988 (GD 165B, Becklin \& Zuckerman 1988; Kirkpatrick et al. 1999), to $960 \mathrm{~K}$ in 1995 (Gl 229B, Nakajima et al. 1995; Geballe et al. 2002), to $800 \mathrm{~K}$ in 2000 (Gl 570D, Burgasser et al. 2000; Geballe et al. 2001), to $650 \mathrm{~K}$ in 2007 (ULAS J0034-00, Warren et al. 2007). Recently, Delorme et al. (2008) have identified a $620 \mathrm{~K}$ field brown dwarf that, along with ULAS J0034-00, shows a suggestion of an ammonia absorption feature in the $H$-band. They note that, if the apparent ammonia feature deepens at lower effective temperatures, then $\sim 600 \mathrm{~K}$ will be a natural break point between the $\mathrm{T}$ and $\mathrm{Y}$ spectral types. In parallel, imaging searches for objects of planetary mass have revealed $2 \mathrm{M} 1207 \mathrm{~b}$ with a mass $\sim 5$ times that of Jupiter (Chauvin et al. 2004, 2005a; Song et al. 2006) or perhaps $\sim 8 M_{\text {J }}$ (Mohanty et al. 2007), along with a handful of substellar companions (Table 1) at or just above the planet/brown dwarf boundary $\left(13.5 M_{\mathrm{J}}\right)$ as defined by the IAU. Published (Masciadri et al. 2005; Luhman et al. 2007a; Kasper et al. 2007a; Lafrenieŕe et al. 2007) and as yet unpublished (Chauvin et al., in preparation; Farihi et al., in preparation; Marois et al., in preparation) imaging searches with adaptive optics (AO) systems on the VLT and on Keck, with the NICMOS camera on HST, and with IRAC on Spitzer, are sensitive to objects with temperatures $<800 \mathrm{~K}$ as well as companion masses as small as a few Jupiters in the case of many target stars.

To set limits on the masses of planets that can be detected at a given separation from a given target star, the standard procedure in most planet-imaging survey papers is to employ a series of Monte Carlo simulations of an ensemble of extrasolar planets around each star. The luminosity of a planet, based on theoretical mass-luminosity-age calculations, at each semimajor axis is compared with the measured minimum detectable brightness in each annulus around a target star. In this way it is possible to determine just how close to any given star a planet of any given mass might be observable. For example, in a sample of 85 nearby young stars, Lafrenière et al. (2007) were sensitive to planets more massive than $2 M_{\mathrm{J}}$ with a projected separation in the range 40-200 AU around a typical target. They found none, and at a $95 \%$ confidence level concluded that at most $12 \%$ of stars harbor a planet more massive than $2 M_{\mathrm{J}}$ between 50 and $295 \mathrm{AU}$. Nielsen et al. (2008) found no planets orbiting 60 stars and concluded with $95 \%$ confidence that the fraction of stars with planets with semimajor axis between 20 and $100 \mathrm{AU}$ and mass above $4 M_{\mathrm{J}}$ is $<20 \%$. Kasper et al. (2007a) derived a frequency of giant planets with masses above $2-3 M_{\mathrm{J}}$ at separations larger than 30 AU around nearby G, K, and M-type stars to be $\leq 5 \%$.

Notwithstanding the above published and unpublished survey sensitivities down to planets of a few Jupiter masses, Table 1 includes all companions reported to date with masses probably less than 20 times that of Jupiter. In the present paper we gather together results from the many papers listed above and from some additional papers to: (1) demonstrate that substellar companions at large semi-major axes (beyond those accessible to the techniques of precision radial velocities and microlensing) can be accounted for in a Jeans-mass fragmentation model; (2) derive a first estimate of the brown dwarf companion initial mass function (IMF) over the entire range of brown dwarf masses; (3) infer that Y dwarfs may be detected occasionally as companions to old stars, but will be particularly rare as companions to stars with ages $<2$ Gyr.

We first consider the significance of Table 1 in the context of formation scenarios for brown dwarfs and massive planets with 
Table 1. Lowest mass companions $\left(M \leq 20 M_{\mathrm{J}}\right)$ imaged to date.

\begin{tabular}{cccccccl}
\hline \hline \multirow{2}{*}{ Object } & \multicolumn{2}{c}{ Sp. Type } & $\begin{array}{c}\text { Age } \\
(\mathrm{Myr})\end{array}$ & $\begin{array}{c}M_{\text {pri }} \\
\left(M_{\odot}\right)\end{array}$ & $\begin{array}{c}M_{\text {sec }} \\
\left(M_{\mathrm{J}}\right)\end{array}$ & $\begin{array}{c}\text { Sep. } \\
(\mathrm{AU})\end{array}$ & Ref. \\
\cline { 2 - 3 } & Primary & Secondary & & \\
\hline 2M1207 & M8 & L5 & 8 & 0.025 & $5^{a}$ & 46 & Chauvin et al. (2004) \\
AB Pic & K2V & L1 & 30 & 0.84 & 14 & 248 & Chauvin et al. (2005c) \\
Oph 11 & M9 & M9.5 & 5 & 0.0175 & 15 & 237 & Close et al. (2007), Luhman et al. (2007b) \\
GQ Lup & K7V & L1.5 & $3 ?$ & 0.7 & 17 & 100 & Neuhauser et al. (2005) \\
HN Peg & G0V & T2.5 & $200^{b}$ & 1.0 & 18 & 795 & Luhman et al. (2007a) \\
TWA 5 & M1.5 & M8/8.5 & 8 & 0.40 & 20 & 98 & Lowrance et al. (1999) \\
LP 261-75 & dM4.5e & L6 & $100-200$ & 0.12 & 20 & 506 & Kirkpatrick et al. (2000) \\
\hline
\end{tabular}

The mass listed for GQ Lup B is the average of the geometric means of the range of likely masses given in McElwain et al. (2007) and Marois et al. (2007).

${ }^{a}$ Mohanty et al. (2007) argue that the mass of $2 \mathrm{M} 1207 \mathrm{~b}$ is $8 \pm 2 M_{\mathrm{J}}$.

${ }^{b}$ We list the age of the HN Peg system as 200 Myr while Luhman et al. (2007a) estimated an age of 300 Myr. We prefer the younger age for the following reasons. The lithium $6708 \AA$ equivalent width is $\sim 105 \mathrm{~m} \AA$ based on an average of independent measurements of 101 and $110 \mathrm{~m} \AA$ by Gaidos et al. (2000) and Chen et al. (2001). With a Johnson $B-V=0.59$ mag, from Hipparcos, or derived from Tycho-2 data (Bessell 2000), Fig. 3 in Zuckerman \& Song (2004) and Fig. 1 in Zuckerman et al. (2006) indicate a lithium age for HN Peg slightly older than that of the Pleiades. The Galactic space motion $U V W(-15,-22,-11$; Nordstrom et al. 2004) is also consistent with those of many young (Pleiades age or younger) stars in the solar vicinity. The logarithmic ratio of X-ray luminosity (as measured in the ROSAT All Sky Survey) to bolometric luminosity is -4.43. This is similar to the Pleiades or the slightly older Carina-Near moving group (see Fig. 2 in Zuckerman et al. 2006). Luhman et al. (2007a) mention chromospheric activity suggestive of an age of 0.35 Gyr. But for a rotation period of 4.91 days (for HN Peg, Gaidos et al. 2000), Song et al. (2004) show that, for youthful stars, CaII emission consistently overestimates stellar ages by a factor of at least a few relative to Li/X-ray/UVW ages. This rotation period and $B-V$ can be used to derive a gyrochronology age of $247 \pm 42 \mathrm{Myr}$ (Barnes 2007). Combination of this age with the somewhat younger ages indicated by lithium, UVW, and X-ray flux, suggests $200 \mathrm{Myr}$ as the most probable age of HN Peg.

semi-major axes sufficiently large for imaging detection with existing instruments. Then we discuss implications of Table 1 for imaging discovery of Y-type substellar companions (Kirkpatrick 2000). This new spectral class, cooler than T-type, may begin to appear at effective temperatures around $500 \mathrm{~K}$ (e.g., Burrows et al. 2003).

\section{Discussion}

\subsection{Fragmentation by gravitational instability}

Thirty years ago, Low \& Lynden-Bell (1976) published a paper "The minimum Jeans mass, or when fragmentation must stop" in which they derived the minimum fragment mass in a typical dark molecular cloud. This mass, about 7 times that of Jupiter, was found to be insensitive to properties of the interstellar dust and cosmic ray heating flux, while various processes (e.g., rotation, magnetic fields, late accretion) would normally be expected to increase this minimum mass. Furthermore, as Low \& Lynden-Bell pointed out, (a) for a fragment to split it must have at least twice the minimum mass and (b) since a Jeans mass perturbation has a zero growth rate, one would expect a real growing perturbation to be somewhat more massive than a Jeans mass. Subsequently, Bate and collaborators (see Bate 2005, and references therein), Boyd \& Whitworth (2005), Padoan \& Nordlund (2004), Padoan et al. (2005), Whitworth \& Stamatellos (2006), and Padoan et al. (2007) considered additional processes such as turbulence, shock compression and the role of magnetic fields and derived fragment masses that could be as low as three times Jupiter's mass.

As indicated in Table 1 and discussed below, at present, no imaged companion is known with a mass clearly below 7 Jupiter masses despite the fact that the imaging searches listed in Sect. 1 should have been sensitive enough to detect some at wide separation if they are copious. Indeed the apparent pile-up of minimum substellar masses at about twice this value (Table 1) is qualitatively consistent with the considerations of Low \& Lynden-Bell outlined in the previous paragraph.
Notwithstanding that AO and HST imaging programs focus on detection of massive planets at the smallest measurable separations, typically $1^{\prime \prime}-2^{\prime \prime}$ or $<50 \mathrm{AU}$, the characteristic separation in the Table 1 binaries is hundreds of AU. Depending on projection effects, even the semi-major axis of $2 \mathrm{M} 1207 \mathrm{~b}$ might be much larger than $46 \mathrm{AU}$. Low \& Lynden-Bell derive an average distance between fragments at last fragmentation of a few $100 \mathrm{AU}$, consistent with the typical separation of pairs listed in Table 1. Rafikov (2005) considered the possibility of giant planet production by gravitational instability in protoplanetary disks. His model can produce massive planets at $\sim 100 \mathrm{AU}$ with masses similar to those listed in Table 1, if the initial disk mass is at least a few tenths of a solar mass ${ }^{1}$. Further consideration of gravitational fragmentation in massive young proto-planetary disks can be found in Stamatellos \& Whitworth (2008).

Objects of planetary mass might exist at large semi-major axes as a consequence of physical mechanisms other than fragmentation. For example, it has been suggested that three body interactions involving a star and either two nearby orbiting planets or a second star with a planet might gravitationally eject a planet to a large semi-major axis. However, between the mass of Jupiter and 14 times this mass, the number of planets discovered by the precision radial velocity technique rises steeply with decreasing mass (Marcy et al. 2005; Lovis et al. 2006). Thus, given that the AO and HST programs listed in Sect. 1 are often sensitive at large separations to planets down to a few Jupiter masses, and that in 3-body interactions one expects the lowest mass

\footnotetext{
1 Rafikov (2005) presents arguments against gravitational instability occurring at 1 and at $10 \mathrm{AU}$, semi-major axes appropriate to the planets discovered by the precision radial velocity (PRV) technique. However, as noted by Chauvin et al. (2005c) and by Rice et al. (2003) inspection of the PRV database indicates that the well-known correlation between high stellar metallicity and the existence of planets may not obtain for stars with the highest mass planets $\left(>7 M_{\mathrm{J}}\right)$. That is, the relatively few highest mass PRV planets may have formed by gravitational collapse, which, compared to core accretion, is relatively insensitive to metallicity.
} 


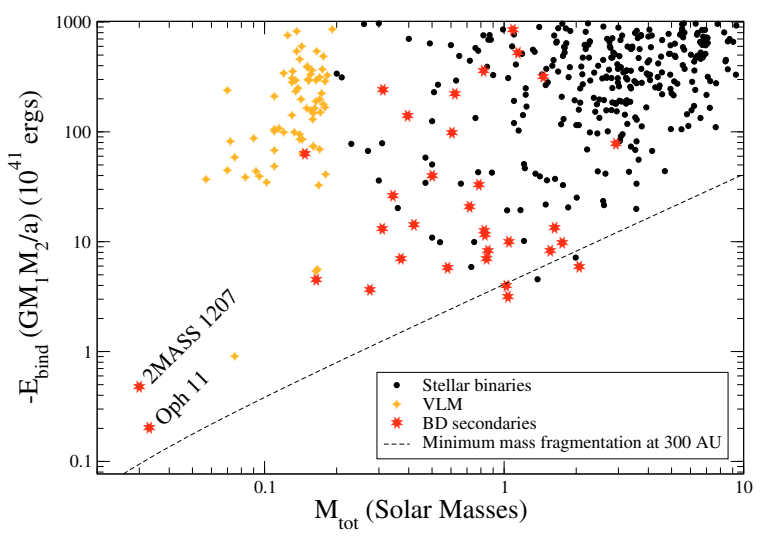

Fig. 1. Binding energies of lowest mass companions known to date. For 2M1207 and Oph 11 both the primary and secondary are substellar. Binaries with stellar primaries are from Fischer \& Marcy (1992) and Tokovinin (1997). Very low mass (VLM) binary data are from Siegler's VLM archive http://paperclip.as . arizona.edu/ nsiegler/VLM_binaries/.

object to be ejected, the distribution of masses given in Table 1 is inconsistent with the ejection model.

\subsection{Y type companions}

The upper temperature limit for a Y-type object is not known; in the following we assume it to be $500 \mathrm{~K}$. Based on Table 1 and on the above considerations, the percentage of stars with companions with large semi-major axes and mass $\lesssim 15$ Jupiter masses appears to be very small indeed. According to Baraffe et al. (2003) and Burrows et al. (2003) the time needed for a 15 Jupiter mass object to cool to $500 \mathrm{~K}$ is $\sim 2$ Gyr. Given the many hundreds of young stars with ages $\lesssim 100 \mathrm{Myr}$ that have been searched with HST and ground-based AO down to this mass, that only three such systems are now known (AB Pic, HN Peg and GQ Lup) indicates that even at $2 \mathrm{Gyr}$, Y-dwarfs should be rare as companions to stars in wide orbits. In other words, for Y-type companions to be abundant at $\sim 2 \mathrm{Gyr}$, many early to mid-L companions ( $\$ 15 M_{\mathrm{J}}$ ) to young ( $\$ 100 \mathrm{Myr}$ ) stars should have been detected in imaging searches of the sort listed in Sect. 1. But such L-type companions are very rare.

The situation regarding Y-type secondaries of $\sim 15$ Jupiter mass where the primary is a brown dwarf is probably even more unfavorable. Although two substellar binaries belonging to very young associations (2M1207 and Oph 11) appear in Table 1, no comparable systems are known to exist among the field brown dwarfs, not even those as young as AB Pic and HN Peg. Indeed, a recent search for wide companions to $132 \mathrm{M} 7-\mathrm{L} 8$ primaries in the field came up completely empty handed (Allen et al. 2007). The low binding energies as displayed in Fig. 1 may be a clue as to why older analogs to 2M1207 and Oph 11 are so rare. However, this connection need not necessarily be straightforward because Burgasser et al. (2003) argue that the deficiency of field brown dwarf binaries with semimajor axes $>10$ AU cannot be explained as due to disruption over Gyrs by encounters with stars and giant molecular clouds. Additional discussion of this point and others may be found in Burgasser et al. (2003, 2006) and in Allen et al. (2007) and Close et al. (2007). If the separation of a minimum mass fragment $\left(\lesssim 15 M_{\mathrm{J}}\right)$ is as large as a few $100 \mathrm{AU}$ as described above, then such fragments will not be found among the population of $10 \mathrm{AU}$ brown dwarf binaries previously discovered with HST and with ground-based AO.

\subsection{IMF for brown dwarf secondaries to stellar primaries}

In Tables 1 and 2 we have gathered from the literature as many brown dwarf secondaries to stellar primaries as we could find, and then plotted their number distribution with mass $(M)$ in Fig. 2. Table 1 contains two systems with brown dwarf primaries, these do not appear in Fig. 2. In comparison with the steep Salpeter distribution for intermediate mass stars - number, $N$, proportional to $\left(M^{-2.35}\right)$ - the Fig. 2 distribution appears much flatter. This figure displays a striking separation in companion mass between youthful and oldish systems. It seems clear that the systems used to detect brown dwarfs around oldish stars have generally been insufficiently sensitive to reveal cool, low mass, brown dwarfs. Therefore, we use only the dozen systems with ages $\$ 300$ Myr to derive the brown dwarf companion IMF; that is, $N$ is proportional to mass as $M^{-1.2 \pm 0.2}$ between 13 and $79 M_{\mathrm{J}}$.

For free-floating low mass objects, Allen et al. (2005) derived $N$ proportional to $\left(M^{-0.3 \pm 0.6}\right)$, which covered masses only down to about $40 M_{\mathrm{J}}$. Allen's sample is the nearby stars. A recent paper by Anderson et al. (2008) focuses instead on the IMF of free-floating low mass stars and brown dwarfs in six very young clusters (1-2 Myr old) and in the Pleiades at distances between 125 and 830 pc from Earth. They probe masses down to about $30 M_{\mathrm{J}}$ and deduce that the mass function is falling as one passes from the stellar to the brown dwarf regime (i.e., in the expression for $(\mathrm{d} N / \mathrm{d} M)$, the exponent on $M$ is positive, rather than negative as found by Allen et al. and in the present paper). With their large error bar $( \pm 0.6)$, the Allen et al. (2005) result is not incompatible with the falling IMF deduced by Anderson et al. (2008).

However, we are troubled by a number of aspects of the Anderson et al. (2008) conclusion. Based on results for the Taurus star-forming region reported by Konopacky et al. (2007), the number of unresolved binaries is apt to be substantially larger than assumed by Anderson et al. (see their Sect. 4). The Konopacky paper is not cited by Anderson et al. In addition, the Anderson et al. analysis implicitly assumes that low mass objects (i.e., brown dwarfs) are fully formed and would be noted at their final masses by a cluster age of 1 or $2 \mathrm{Myr}$. This assumption may not be valid.

Both Allen et al. (2005) and Anderson et al. (2008) are analyzing the IMF for low mass, free-floating, objects whereas our concern is the IMF for brown dwarf companions to stars. Burgasser et al. (2007) consider the mass and mass distribution of companions to late-F to K-type dwarfs within 25 pc of Earth. Their Fig. 1 suggests that, the wider the binary, the closer the companion mass function approaches the canonical field distribution. Thus, the companion IMF depicted in our Fig. 2, being based on wide separation binaries, might mimic the field IMF. Because almost all of the secondaries in young binaries upon which Fig. 2 is based have masses below the low mass cutoff of the Allen et al. and Anderson et al. studies, a direct comparison of our IMF with theirs' is not possible.

Concerning only companions (rather than field objects), it is of interest to know how the brown dwarf companion mass function matches onto the very low mass stellar companion mass function. The latter is a complicated issue (Burgasser et al. 2007; Reid 2008, personal communication), and, to the best of our knowledge, there is no published companion mass function that encompasses secondary masses that straddle the stellar/substellar boundary. For example, notwithstanding their interest in low mass companions, in the second edition of their 
Table 2. Brown dwarf secondaries $\left(M \geq 25 M_{\mathrm{J}}\right)$ to stellar primaries.

\begin{tabular}{|c|c|c|c|c|c|c|c|}
\hline \multirow[t]{2}{*}{$\overline{\overline{\text { Object }}}$} & \multicolumn{2}{|c|}{ Sp. Type } & \multirow{2}{*}{$\begin{array}{c}\text { Age } \\
(\mathrm{Myr})\end{array}$} & \multirow{2}{*}{$\begin{array}{l}M_{\text {pri }} \\
\left(M_{\odot}\right)\end{array}$} & \multirow{2}{*}{$\begin{array}{l}M_{\mathrm{sec}} \\
\left(M_{\mathrm{J}}\right)\end{array}$} & \multirow{2}{*}{$\begin{array}{l}\text { Sep. } \\
\text { (AU) }\end{array}$} & \multirow[t]{2}{*}{ Ref. } \\
\hline & Primary & Secondary & & & & & \\
\hline GJ 802 & $\overline{\mathrm{M} 5+\mathrm{M} 5}$ & L6 & $\sim 2000$ & $0.28^{*}$ & 66 & 1.46 & Ireland et al. (2008) \\
\hline SCR1845-6357 & M8.5 & T6 & $1800-3100$ & 0.1 & 45 & 4 & Kasper et al. (2007b) \\
\hline Gl 337 & $\mathrm{G} 8+\mathrm{K} 1$ & L8 & $600-3400$ & 1.74 & $110^{*}$ & 11 & Wilson et al. (2001) \\
\hline G $124-62$ & $\mathrm{dM} 4.5 \mathrm{e}$ & L0.5 & $500-800$ & 0.24 & $72 *$ & 13 & Martin et al. (1999) \\
\hline Gl 779B & G1V & L4.5 & $1000-3000$ & 1.02 & 66 & 13 & Liu et al. (2002) \\
\hline 2MASS J1707-05 & M9 & L3 & $500-5000$ & 0.077 & 70 & 15 & McElwain \& Burgasser (2006) \\
\hline Gl 86 & $\mathrm{~K} 1 \mathrm{~V}$ & $\mathrm{~L} / \mathrm{T}$ & 1000-9999 & 0.77 & 50 & 19 & Els et al. (2001) \\
\hline G 239-25 & M1.5 & L0 & & 0.32 & $75 ?$ & 30 & Golimowski et al. (2004) \\
\hline HD 49197 & F5 & L4 & $260-790$ & 1.4 & 54 & 42 & Metchev \& Hillenbrand (2004) \\
\hline Gl 229 & $\mathrm{M} 1 / 2 \mathrm{~V}$ & $\mathrm{~T} 7$ & 3000 & 0.56 & 35 & 45 & Nakajima et al. (1995) \\
\hline HD 130948 & G2V & $\mathrm{L} 4+\mathrm{L} 4$ & $<800$ & 1.00 & $140^{*}$ & 47 & Potter et al. (2002) \\
\hline GL 569 B & $\mathrm{M} 2.5 \mathrm{~V}$ & & $250-500$ & 0.50 & $123^{*}$ & 49 & Lane et al. (2001) \\
\hline GJ 1001 & M3.5 & L5 & $>1000$ & 0.4 & $100 *$ & 178 & Goldman et al. (1999) \\
\hline HR 7329 & A0Vn & M7/8 & 12 & 2.90 & 30 & 198 & Lowrance et al. (2000) \\
\hline GG Tau B & M5 & M7 & $1-2$ & 0.12 & 44 & 207 & White et al. (1999) \\
\hline LHS 5166 & $\mathrm{dMe} 4.5$ & L4 & $<2600$ & 0.24 & 70 & 228 & Seifahrt et al. (2005) \\
\hline GJ 1048 & $\mathrm{~K} 3 \mathrm{~V}$ & L1 & 1000 & 0.72 & 65 & 250 & Gizis et al. (2001) \\
\hline GSC 8047-232 & $\mathrm{K} 3 \mathrm{~V}$ & L0 & $10-50$ & 0.80 & 25 & 279 & Chauvin et al. (2005b) \\
\hline G 196-3 & $\mathrm{dM} 3 \mathrm{Ve}$ & L2 & 100 & 0.25 & 25 & 300 & Rebolo et al. (1998) \\
\hline DH Tau & $\mathrm{M} 0.5 \mathrm{~V}$ & L2 & $0.1-4$ & 0.33 & ? & 330 & Itoh et al. (2005) \\
\hline ScoPMS214 & K1IV & M6 & 5 & 1.02 & 25 & 450 & Metchev (2006) \\
\hline HD 3651 & K0V & $\mathrm{T} 7.5$ & 1000-9999 & 0.79 & 40 & 480 & Mugrauer et al. (2006) \\
\hline HD 203030 & G8V & L7.5 & $130-400$ & 1.60 & 23 & 487 & Metchev \& Hillenbrand (2006) \\
\hline GJ 618.1 & MOV & L2.5 & $500-12000$ & 0.51 & 70 & 1089 & Wilson et al. (2001) \\
\hline$\epsilon$ Indi $\mathrm{Ba}+\mathrm{b}$ & $\mathrm{K} 4.5 \mathrm{Ve}$ & $\mathrm{T} 1+\mathrm{T} 6$ & $800-2000$ & 0.77 & $75^{*}$ & 1459 & Scholz et al. (2003) \\
\hline Gliese 570 & $\mathrm{~K} 5+\mathrm{M} 1+$ & $\mathrm{T} 7$ & 2000-9999 & $1.7 *$ & 50 & 1526 & Burgasser et al. (2000) \\
\hline Gl 417 & G0V & L4.5 & $80-300$ & 1.0 & 35 & 1955 & Kirkpatrick et al. (2000) \\
\hline HD 89744 & F7V & L0 & $1500-3000$ & 1.48 & 78 & 2460 & Wilson et al. (2001) \\
\hline Gl 584 & $\mathrm{G} 2+\mathrm{G} 2$ & L8 & $1000-2500$ & $2.0 *$ & 60 & 3612 & Kirkpatrick et al. (2000) \\
\hline
\end{tabular}

For multiple systems consisting of a close brown dwarf binary to a stellar primary, total mass of the brown dwarf binary is listed in the table as $M_{\text {sec }}$ with “*” mark. Likewise, when the primary is a multiple system, the total mass of stars is listed as $M_{\text {pri }}$.

book, Reid \& Hawley (2005) declined to address the shape of the companion mass function near the stellar/substellar boundary.

In a paper that slightly postdated the Reid/Hawley book, Farihi et al. (2005) derived the companion mass function across the stellar/substellar boundary for white dwarf primaries, i.e. stars that, when on the main sequence, were on average more massive than the Sun. Farihi et al. found that late M-type (i.e., minimum mass stellar companion) are uncommon compared to mid M-type companions (see their Fig. 6). Their survey of 261 white dwarfs capable of detecting companions at orbital separations between $\sim 100$ and $5000 \mathrm{AU}$ with masses as low as $50 M_{\mathrm{J}}$ (corresponding to the rightmost mass bin of our Fig. 2) found no brown dwarf companion. Therefore, with the caveats that conclusions based on young companions plotted in Fig. 2 suffer from small number statistics, and the Farihi et al. sample is limited to medium mass primaries, it appears that companions with masses just above or below the brown dwarf/stellar dividing line are rare indeed. Recent model simulations of the formation of brown dwarfs and very low mass stars are consistent with this conclusion (Stamatellos \& Whitworth 2008). Figures 5 and 13 of Stamatellos \& Whiteworth (2008) illustrate that both very low mass stars and brown dwarfs, at the few hundred AU semimajor axes of interest in the present paper, are expected to be uncommon as secondaries to solar mass stars.

\section{Conclusions}

We have gathered from the literature those binary systems with imaged companions of the least mass (Table 1 and Fig. 2).
Given the very large number of target stars and brown dwarfs observed in imaging ground- and space-based searches for low mass brown dwarfs and high mass planets, these Table 1 objects represent pretty slim pickings. We show that minimum Jeans mass fragmentation of an interstellar molecular cloud, as described a long time ago (Low \& Lynden-Bell 1976), can account for these data at least as well as any more recent model for the production of brown dwarfs. Similarly, gravitational instability in massive protoplanetary disks (Rafikov 2005; Stamatellos \& Whitworth 2008) might account for some/many of the observed systems.

To derive the IMF for brown dwarf secondaries to stellar primaries it is essential to consider only youngish systems because data presented in this paper show that telescope/detector sensitivities are often insufficient to detect old, low mass, brown dwarfs. We find that the number of brown dwarf companions is proportional to mass as $M^{-1.2 \pm 0.2}$ down to the bottom of the brown dwarf mass range, $\sim 13$ Jupiter masses. While this power law index might not apply to free-floating field brown dwarfs, the precipice in the companion mass function for masses below $13 M_{\mathrm{J}}$, suggests that free floating objects with masses in the planetary range will be rare.

The extreme rarity of imaged companions below $\sim 15$ Jupiter masses suggests that Y-type objects $\left(T_{\text {eff }} \lesssim 500 \mathrm{~K}\right)$ will be imaged as companions to very few, if any, stars with ages $<2$ Gyr. Even for a star system as old as $7 \mathrm{Gyr}$, according to the models of Baraffe et al. (2003), a brown dwarf would have to be less massive than $\sim 25$ Jupiter masses to cool to $500 \mathrm{~K}$. Imaging discovery of a Y-type companion to a substellar primary is even 


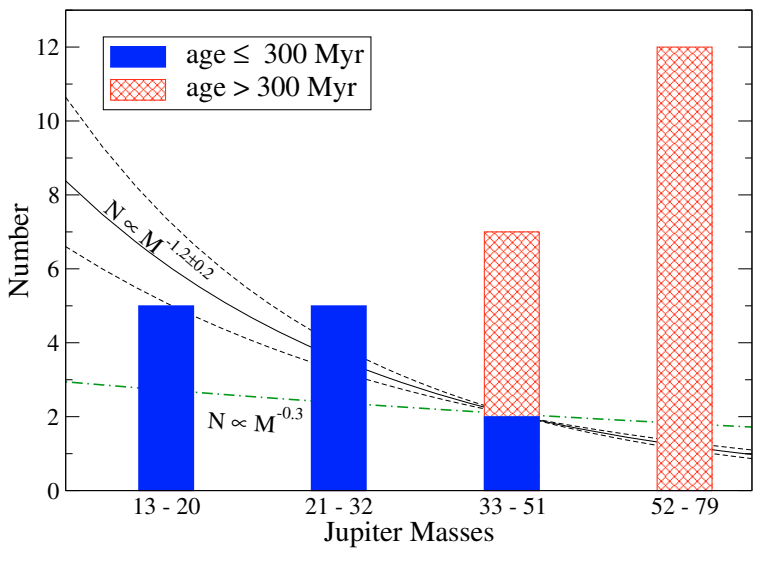

Fig. 2. Histogram of masses of secondaries with stellar primaries. Data are from Tables 1 and 2 of this paper. For stellar primaries with brown dwarf secondaries that are themselves compact binaries (e.g., GL 337B, G 124-62B, etc.), we treat these compact binaries as singles by plotting total masses. The brown dwarf mass distribution is certainly flatter than that of Salpeter $\left(N \propto M^{-2.35}\right)$. Considering only systems with ages $\leq 300 \mathrm{Myr}$ (see Sect. 2.3), the number of secondaries is proportional to about $M^{-1.2}$. As described in the text and displayed in Table 1, there is a sharp secondary mass cutoff near 15 Jupiter masses - that is, for stellar primaries, no imaged secondaries with masses below 13 Jupiter masses are known. The dot-dashed line indicates the brown dwarf mass distribution expected if the result for free-floating objects ( $N$ proportional to $\left.M^{-0.3 \pm 0.6}\right)$ derived by Allen et al. (2005) for masses $>40 M_{\mathrm{J}}$ obtains all the way down to $13 M_{\mathrm{J}}$.

less likely, at any age, given the absence of wide companions to field brown dwarfs (e.g., Allen et al. 2007). Thus, all in all, Y-type secondaries should appear in imaging programs only infrequently. Given the hundreds of young stars surveyed in the planet hunting programs listed in the Introduction and the number of low mass brown dwarfs indicated in Fig. 2, for a well choosen set of old stars, perhaps one in 100 might be orbited at large separations by a Y dwarf.

Acknowledgements. We thank B. Hansen, M. Jura, and Neill Reid for helpful suggestions and the referee for constructive comments. This research was supported in part by a NASA grant to UCLA.

Note added in proof. Dr. S. Metchev has called to our attention three recent papers (Kouwenhoven et al. 2007, A\&A, 474, 77; Kraus et al. 2008, ApJ, 679, 762; Metchev \& Hillenbrand 2008, ApJS, in press) of potential relevance to Sect. 2.3 and Fig. 2 of the present paper. These three papers do address the form of the companion mass function across the stellar/substellar boundary and into the brown dwarf mass range. However, in comparison with our study that encompasses field stellar primaries of all spectral types and of origin in a wide variety of environments, the three other studies are more limited; either they are confined to a single region of star formation or to a limited range of primary masses or both. Because of such differences, IMFs for brown dwarf secondaries to stellar primaries derived in the various papers may not be inconsistent with each other. For additional details, please see the note added in proof in the Metchev $\&$ Hillenbrand (2008) paper.

\section{References}

Allen, P. R., Koerner, D. W., Reid, I. N., \& Trilling, D. E. 2005, ApJ, 625, 385 Allen, P. R., Koerner, D. W., McElwain, M. W., Cruz, K. L., \& Reid, I. N. 2007, AJ, 133, 971

Anderson, M., Meyer, M. R., Greissl, J., \& Aversa, A. 2008 [arXiv:0807.1354v1]
Baraffe, I., Chabrier, G., Barman, T. S., Allard, F., \& Hauschildt, P. H. 2003, A\&A, 402, 701

Barnes, S. A. 2007, ApJ, 669, 1167

Bate, M. R. 2005, MNRAS, 363, 363

Becklin, E. E., \& Zuckerman, B. 1988, Nature, 336, 656

Bessell, M. S. 2000, PASP, 112, 961

Boyd, D. F. A., \& Whitworth, A. P. 2005, A\&A, 430, 1059

Burgasser, A. J., Kirkpatrick, J. D., Cutri, R. M., et al. 2000, ApJ, 531, L57

Burgasser, A. J., Kirkpatrick, J. D., Reid, I. N., et al. 2003, ApJ, 586, 512

Burgasser, A. J., Kirkpatrick, J. D., Cruz, K. L., et al. 2006, ApJS, 166, 585

Burgasser, A. J., Reid, I. N., Siegler, N., et al. 2007, in Protostars and Planets V,

ed. B. Reipurth, D. Jewitt, \& K. Keil (Tucson: University of Arizona Press), 427

Burrows, A., Sudarsky, D., \& Lunine, J. I. 2003, ApJ, 596, 587

Chauvin, G., Lagrange, A.-M., Dumas, C., et al. 2004, A\&A, 425, L29

Chauvin, G., Lagrange, A.-M., Dumas, C., et al. 2005a, A\&A, 438, L25

Chauvin, G., Lagrange, A.-M., Lacombe, F., et al. 2005b, A\&A, 430, 1027

Chauvin, G., Lagrange, A.-M., Zuckerman, B., et al. 2005c, A\&A, 438, L29

Chen, Y. Q., Nissen, P. E., Benoni, T., \& Zhao, G. 2001, A\&A, 371, 943

Close, L. M., Zuckerman, B., Song, I., et al. 2007, ApJ, 660, 1492

Delorme, P., Croll, B., Matthews, J. M., et al. 2008, A\&A, 482, 961

Els, S. G., Sterzik, M. F., Marchis, F., et al. 2001, A\&A, 370, L1

EROS Collaboration, et al. 1999, A\&A, 351, L5

Farihi, J., Becklin, E. E., \& Zuckerman, B. 2005, ApJS, 161, 394

Fischer, D. A., \& Marcy, G. W. 1992, ApJ, 396, 178

Gaidos, E. J., Henry, G. W., \& Henry, S. M. 2000, AJ, 120, 1006

Geballe, T. R., Saumon, D., Leggett, S. K., et al. 2001, ApJ, 556, 373

Geballe, T. R., Knapp, G. R., Leggett, S. K., et al. 2002, ApJ, 564, 466

Gizis, J. E., Kirkpatrick, J. D., \& Wilson, J. C. 2001, AJ, 121, 2185

Golimowski, D. A., Henry, T. J., Krist, J. E., et al. 2004, AJ, 128, 1733

Ireland, M. J., Kraus, A., Martinache, F., Lloyd, J. P., \& Tuthill, P. G. 2008, ApJ, in press [arXiv: 0801.1525$]$

Itoh, Y., Hayashi, M., Tamura, M., et al. 2005, ApJ, 620, 984

Kasper, M., Apai, D., Janson, M., \& Brandner, W. 2007a, A\&A, 472, 321

Kasper, M., Biller, B. A., Burrows, A., et al. 2007b, A\&A, 471, 655

Kirkpatrick, J. D. 2000, From Giant Planets to Cool Stars, ASP Conf. Ser., 212, 20

Kirkpatrick, J. D., Allard, F., Bida, T., et al. 1999, ApJ, 519, 834

Kirkpatrick, J. D., Reid, I. N., Liebert, J., et al. 2000, AJ, 120, 447

Konopacky, Q. M., Ghez, A. M., Rice, E. L. \& Duchene, G. 2007, ApJ, 663, 394

Lafrenière, D., Reid, I. N., Liebert, J., et al. 2007, ApJ, 670, 1367

Lane, B. F., Zapatero Osorio, M. R., Britton, M. C., Martín, E. L., \& Kulkarni, S. R. 2001, ApJ, 560, 390

Liu, M. C., Fischer, D. A., Graham, J. R., et al. 2002, ApJ, 571, 519

Lovis, C., et al. 2006, Proc. SPIE, 6269

Low, C., \& Lynden-Bell, D. 1976, MNRAS, 176, 367

Lowrance, P. J., McCarthy, C., Becklin, E. E., et al. 1999, ApJ, 512, L69

Lowrance, P. J., Schneider, G., Kirkpatrick, J. D., et al. 2000, ApJ, 541, 390

Luhman, K. L., Patten, B. M., Marengo, M., et al. 2007a, ApJ, 654, 570

Luhman, K. L., Allers, K. N., Jaffe, D. T., et al. 2007b, ApJ, 659, 1629

Marcy, G., Butler, R. P., Fischer, D., et al. 2005, Progr. Theor. Phys. Suppl., 158, 24

Marois, C., Macintosh, B., \& Barman, T. 2007, ApJ, 654, L151

Masciadri, E., Mundt, R., Henning, T., Alvarez, C., \& Barrado y Navascués, D. 2005, ApJ, 625, 1004

McElwain, M. W., \& Burgasser, A. J. 2006, AJ, 132, 2074

McElwain, M. W., Metchev, S. A., Larkin, J. E., et al. 2007, ApJ, 656, 505

Metchev, S. A. 2006, Ph.D. Thesis, California Institute of Technology

Metchev, S. A., \& Hillenbrand, L. A. 2004, ApJ, 617, 1330

Metchev, S. A., \& Hillenbrand, L. A. 2006, ApJ, 651, 1166

Mohanty, S., Jayawardhana, R., Huelamo, N., \& Mamajek, E. 2007, ApJ, 657, 1064

Mugrauer, M., Seifahrt, A., Neuhäuser, R., \& Mazeh, T. 2006, MNRAS, 373, L31

Nakajima, T., Oppenheimer, B. R., Kulkarni, S. R., et al. 1995, Nature, 378, 463

Neuhauser, R., Guenther, E. W., Wuchterl, G., et al. 2005, A\&A, 435, L13

Nielsen, E. L., Close, L. M., Biller, B. A., Masciadri, E., \& Lenzen, R. 2008, ApJ, 674, 466

Nordström, B., Mayor, M., Andersen, J., et al. 2004, A\&A, 418, 989

Padoan, P., \& Nordlund, A. 2004, ApJ, 617, 559

Padoan, P., Kritsuk, A., Norman, M. L., \& Nordlund, Å. 2005, ApJ, 622, L61

Padoan, P., Nordlund, Å, Kritsuk A., Norman, M., \& Li, P. S. 2007, ApJ, 661, 972

Potter, D., Martín, E. L., Cushing, M. C., et al. 2002, ApJ, 567, L133

Rafikov, R. R. 2005, ApJ, 621, L69

Rebolo, R., Zapatero Osorio, M. R., Madruga, S., et al. 1998, Science, 282, 1309 
Reid, I. N., \& Hawley, S. 2005, New Light on Dark Stars, second edition (Chichester, UK: Springer)

Rice, W. K. M., Armitage, P. J., Bonnell, I. A., et al. 2003, MNRAS, 346, L36

Scholz, R.-D., McCaughrean, M. J., Lodieu, N., \& Kuhlbrodt, B. 2003, A\&A, 398, L29

Seifahrt, A., Mugrauer, M., Wiese, M., Neuhäuser, R., \& Guenther, E. W. 2005, Astron. Nachr., 326, 974

Song, I., Zuckerman, B., \& Bessell, M. S. 2004, ApJ, 614, L125

Song, I., Schneider, G., Zuckerman, B., et al. 2006, ApJ, 652, 724
Stamatellos, D., \& Whitworth, A. P. 2008, MNRAS, in press [arXiv: 0810.1687]

Tokovinin, A. A. 1997, A\&AS, 124, 75

Warren, S., Mortlock, D. J., Leggett, S. K., et al. 2007, MNRAS, 381, 1400

Wilson, J. C., Kirkpatrick, J. D., Gizis, J. E., et al. 2001, AJ, 122, 1989

White, R. J., Ghez, A. M., Reid, I. N., \& Schultz, G. 1999, ApJ, 520, 811

Whitworth, A. P., \& Stamatellos, D. 2006, A\&A, 458, 817

Zuckerman, B., \& Song, I. 2004, ARA\&A, 42, 685

Zuckerman, B., Bessell, M. S., Song, I., \& Kim, S. 2006, ApJ, 649, L115 